

\title{
Co-movements between Latin American and U.S. stock markets: convergence after the financial crisis?
}

\author{
Andrés Ramírez Hassan* Javier Pantoja Robayo ${ }^{\dagger}$
}

October 29, 2013

\begin{abstract}
Currently, the world is facing a continuous process of integration in different aspects and financial markets are no exception to this development. Despite the fact that global integration is gradual, one can find some specific events that might help to accelerate this trend. This paper shows that after the financial crisis of 2008, which mainly occurred in the United States, the Latin American stock markets exhibit a higher level of convergence, measured by the correlation between the annual returns of their stock market indices. Additionally, we find convergence in the coefficient of sensitivity between Latin American and U.S. stock markets, using dynamic linear models at the regional level. In particular, we uncover consistent movements in the levels of sensitivity between the daily annual returns of the Latin American indices and the S\&P index after the financial crisis. This kind of convergence might be a positive sign to accelerate the integration process in Latin America stock markets, which has had a slow development since its beginning a few years ago.
\end{abstract}

JEL Classification: G01, G15.

Keywords: Dynamic Linear Models, Latin American Stock Markets.

Acknowledgements: We are grateful for the excellent research assistance from David Arango.

${ }^{*}$ Departament of Economics, Universidad EAFIT. aramir21@eafit.edu.co

${ }^{\dagger}$ Departament of Finance, Universidad EAFIT. jpantoja@eafit.edu.co 


\section{Introduction}

Globalization is a trend characterized by the integration process of markets, which can be unequal across different regions and sectors. For example in financial markets globalization has had an enormous effect, especially in developed countries, although this process has been less forceful in emerging economies.

Market integration is a gradual phenomenon that depends on specific circumstances related to geographical, social, cultural, political, and economic issues. However, there are some specific events that may accelerate this process in some regions. Today, a major disturbance in an international market is transmitted in seconds to stock exchanges throughout the world. According to Allen et al. (2012), emerging markets have a market-based financial system, which implies that those markets are affected by financial crises and require more time to recover from economic downturns, such as the subprime crisis in 2008. A crisis like this represents a valuable opportunity to study the volatility of co-movements among U.S. and emerging markets.

Since the liberalization of emerging markets in the late 1980s and early 1990s, those markets have shown a greater interdependence between associated financial markets, economic and financial integration, increasing mobility of portfolio flows, and flow of information between countries. Other studies have shown co-movements between assets and stock markets in different regions (Chen et al., 2002; Sakthivel et al., 2012; Weber, 2013). However to our knowledge, clear results have not been produced for the Latin American financial markets up to now. In particular, Sakthivel et al. (2012) use a bivariate GARCH model to study the transmission of volatility of returns of stock indices in developed countries and India, finding evidence about convergence and volatility transmission between these markets. Fayyad and Daly (2011) employ BEKK MGARCH models to determine the relationship between the volatility of emerging markets and developed markets. Specifically, U.S. and UK versus the Arab League in the first case and European markets in the second, these authors find evidence in both cases. Lee and Rui (2004) use multivariate EGARCH models in two stages to find evidence for volatility transmission from the NASDAQ to Asian emerging markets. Additionally, Liow (2009) establishes market integration as a time-varying process, and the evolution of the pattern of integration 
will be characterized by dynamic changes in the correlation matrix, both at the local and global level. Furthermore, Liow (2009) finds statistical evidence of co-movements in the Asian stock market, which shows empirically the transmission between financial markets around the word, especially between developed and emerging markets. As a consequence, the integration of financial markets in a specific region of the world becomes easier given highly correlated markets, indicating that domestic assets are impacted by similar events in a global scenario.

Financial market integration can contribute positively to the improvement of investor diversification, hedging and leverage. Therefore, this can lead to a process of synchronization, allowing investors to incorporate information in their decision-making process. For instance, an investor could implement hedging strategies using instruments from different domestic markets. Additionally, market integration is an important aspect for global investors when understanding the level of informational efficiency in the international financial architecture. These are some of the reasons that explain why a financial integration process called Mercado Integrado Latino Americano (MILA) began with countries like Chile, Colombia and Peru.

This paper aims to quantify how the level of co-movements between the main Latin American financial markets changed after the financial crisis in United States in 2008. Given that financial markets are dynamic, we use dynamic linear models to evaluate the co-movements between the Latin American and U.S, stock markets. Further, we employ descriptive statistics from dynamic coefficients to show how the level of synchronization of Latin American markets have changed after the financial crisis. We use daily data of the most important stock market indices in Latin America and U.S. from July 2003 and June 2012. Our findings suggest a higher level of co-movements in the Latin American stock markets after the financial crisis. This suggests a greater level of synchronization in the region which reflects a better environment for market integration (Bracker and Koch, 1999). In addition, our work shows evidence about different levels of sensitivity that Latin American countries faced with U.S. market measured with the S\&P Index. For instance, Colombia, Peru and Chile exhibit the smallest sensitivity in comparison with Brazil and Mexico. 
The paper proceeds as follows. Section 2 provides an overview of the Dynamic Linear Models used to check convergence in Latin American and United States stock markets. Section 3 summarizes econometric results. Finally, Section 4 contains some concluding remarks.

\section{Dynamic Linear Models}

This section follows from Petris et al. (2007). State space models consider a time series as the output of a dynamic system perturbed by random disturbances where computations can be implemented using recursive algorithms.

Formally, a state space model consists of an $R^{p}$-valued time series $\left(\theta_{t}: t=0,1, \ldots\right)$ and an $R^{m}$-valued time series $\left(Y_{t}: t=1,2, \ldots\right)$, satisfying the following assumptions:

- $\theta_{t}$ is a Markov chain.

- Conditionally on $\theta_{t}, Y_{t}$ 's are independent and $Y_{t}$ depends on $\theta_{t}$ only.

The consequence of these assumptions is that a state space model is completely specified by the initial distribution $\pi\left(\theta_{0}\right)$ and the conditional densities $\pi\left(\theta_{t} \mid \theta_{t-1}\right)$ and $\pi\left(y_{t} \mid \theta_{t}\right), t \geq 1$.

An important class of state space models is given by Gaussian linear state space models, which is also called dynamic linear models. This model is specified by a Normal prior distribution for the $p$-dimensional state vector at time $t=0$,

$$
\theta_{0} \sim \mathcal{N}_{p}\left(m_{0}, C_{0}\right)
$$

together with a pair of equations for each time $t \geq 1$,

$$
Y_{t}=F_{t} \theta_{t}+v_{t}, v_{t} \sim \mathcal{N}_{m}\left(0, V_{t}\right)
$$

and

$$
\theta_{t}=G_{t} \theta_{t-1}+w_{t}, w_{t} \sim \mathcal{N}_{p}\left(0, W_{t}\right)
$$


where $G_{t}$ and $F_{t}$ are known matrices of order $p \times p$ and $m \times p$, respectively, and $\left(v_{t}\right)_{(t \geq 1)}$ and $\left(w_{t}\right)_{(t \geq 1)}$ are two independent sequences. Equation (2) is called the observation equation, while (3) is the state equation. In this case, $\pi\left(\theta_{t} \mid \theta_{t-1}\right) \sim \mathcal{N}_{p}\left(G_{t} \theta_{t-1}, W_{t}\right)$ and $\pi\left(Y_{t} \mid \theta_{t}\right) \sim \mathcal{N}_{m}\left(F_{t} \theta_{t}, V_{t}\right)$.

Kalman filter: Given the dynamic linear model defined by equation (1), (2) and (3), let $\pi\left(\theta_{t-1} \mid y_{1: t-1}\right) \sim \mathcal{N}_{p}\left(m_{t-1}, C_{t-1}\right)$, then the following statements hold:

- The one step ahead predictive distribution of $\theta_{t}$ given $y_{1: t-1}$ is Gaussian, with parameters

$$
a_{t}=E\left(\theta_{t} \mid y_{1: t-1}\right)=G_{t} m_{t-1}
$$

and

$$
R_{t}=\operatorname{Var}\left(\theta_{t} \mid y_{1: t-1}\right)=G_{t} C_{t-1} G_{t}^{\prime}+W_{t}
$$

- The one step ahead predictive distribution of $Y_{t}$ given $y_{1: t-1}$ is Gaussian, with parameters

$$
f_{t}=E\left(Y_{t} \mid y_{1: t-1}\right)=F_{t} a_{t}
$$

and

$$
Q_{t}=\operatorname{Var}\left(Y_{t} \mid y_{1: t-1}\right)=F_{t} R_{t} F_{t}^{\prime}+V_{t}
$$

- The filtering distribution of $\theta_{t}$ given $y_{1: t}$ is Gaussian, with parameters

$$
m_{t}=E\left(\theta_{t} \mid y_{1: t}\right)=a_{t}+R_{t} F_{t}^{\prime} Q_{t}^{-1} e_{t}
$$

and

$$
C_{t}=\operatorname{Var}\left(\theta_{t} \mid y_{1: t}\right)=R_{t}-R_{t} F_{t}^{\prime} Q_{t}^{-1} F_{t} R_{t}
$$

where $e_{t}=Y_{t}-f_{t}$ is the forecast error.

Kalman smoother: Given the dynamic linear model defined by equation (1), (2) and (3), 
if $\pi\left(\theta_{t+1} \mid y_{1: T}\right) \sim \mathcal{N}_{p}\left(s_{t+1}, S_{t+1}\right)$, then $\pi\left(\theta_{t} \mid y_{1: T}\right) \sim \mathcal{N}_{p}\left(s_{t}, S_{t}\right)$, where

$$
s_{t}=m_{t}+C_{t} G_{t+1}^{\prime} R_{t+1}^{-1}\left(s_{t+1}-a_{t+1}\right)
$$

and

$$
S_{t}=C_{t}-C_{t} G_{t+1}^{\prime} R_{t+1}^{-1}\left(R_{t+1}-S_{t+1}\right) R_{t+1}^{-1} G_{t+1} C_{t}
$$

Kalman forecast: Given the dynamic linear model defined by equation (1), (2) and (3), let $a_{t}(0)=m_{t}$ and $R_{t}(0)=C_{t}$. Then, for $k \geq 1$, the following statements hold.

- The distribution of $\theta_{t+k}$ given $y_{1: t}$ is Gaussian, with

$$
a_{t}(k)=G_{t+k} a_{t, k-1}
$$

and

$$
R_{t}(k)=G_{t+k} R_{t, k-1} G_{t+k}^{\prime}+W_{t+k}
$$

- The distribution of $Y_{t+k}$ given $y_{1: t}$ is Gaussian, with

$$
f_{t}(k)=F_{t+k} a_{t}(k),
$$

and

$$
Q_{t}(k)=F_{t+k} R_{t}(k) F_{t+k}^{\prime}+V_{t}
$$

A dynamic simple linear regression is a particular case of a dynamic linear model such that,

$$
Y_{t}=\left[\begin{array}{ll}
1 & x_{t}
\end{array}\right]\left[\begin{array}{l}
\beta_{1, t} \\
\beta_{2, t}
\end{array}\right]+v_{t}
$$

and

$$
\left[\begin{array}{l}
\beta_{1, t} \\
\beta_{2, t}
\end{array}\right]=\left[\begin{array}{ll}
1 & 0 \\
0 & 1
\end{array}\right]\left[\begin{array}{l}
\beta_{1, t-1} \\
\beta_{2, t-1}
\end{array}\right]+\left[\begin{array}{l}
w_{1, t} \\
w_{2, t}
\end{array}\right],
$$


where $v_{t} \stackrel{i i d}{\sim} \mathcal{N}\left(0, \sigma_{v}^{2}\right)$ and $\left[\begin{array}{l}w_{1, t} \\ w_{2, t}\end{array}\right] \sim \mathcal{N}\left(\left[\begin{array}{l}0 \\ 0\end{array}\right],\left[\begin{array}{cc}\sigma_{w_{1}}^{2} & 0 \\ 0 & \sigma_{w_{2}}^{2}\end{array}\right]\right)$.

\section{Econometric Results}

We use daily data of the most important stock market indices in Latin America and The U.S from July 15, 2003 to June 25, 2012. Specifically, we calculate annual returns of the stock market indices of the following countries: Argentina, Brazil, Chile, Colombia, Mexico, Peru, and the S\&P index from the U.S.

Table (1) presents correlation coefficients in the period 2003-2012 between the S\&P and the Argentine, Brazilian, Chilean, Colombian, Mexican, and Peruvian indices, which are 0.76, 0.70, $0.81,0.62,0.88$ and 0.69 , respectively. These levels of correlation can be considered high. However, if we compare the correlation coefficient before and after the U.S. financial crisis, which initiated in January 2008, we find that the high level of co-movements between these markets is due to the latter period. In fact the correlation coefficient for each country in the period 2008-2012 is higher than the coefficients in the period 2003-2007. This outcome can be seen in Figure (1) where the pattern that follows the Latin American stock market returns resembles in a better way the trajectory of S\&P returns after the U.S. financial crisis. ${ }^{1}$

Table 1: Descriptive statistics: Annual rate of return, 2003-2012.

\begin{tabular}{lccccccc}
\hline Country & Mean & Standard Deviation & Maximum & Minimum & $\begin{array}{c}\text { Correlation } \\
(2003-2012)\end{array}$ & $\begin{array}{c}\text { Correlation } \\
(2003-2007)\end{array}$ & $\begin{array}{c}\text { Correlation } \\
(2008-2012)\end{array}$ \\
\hline Argentine & $25.9 \%$ & $49.5 \%$ & $172.7 \%$ & $-64.5 \%$ & 0.76 & 0.60 & 0.88 \\
Brazil & $39.5 \%$ & $51.8 \%$ & $196.7 \%$ & $-62.3 \%$ & 0.70 & 0.66 & 0.68 \\
Chile & $26.6 \%$ & $31.9 \%$ & $103.4 \%$ & $-51.3 \%$ & 0.81 & 0.72 & 0.81 \\
Colombia & $42.5 \%$ & $45.7 \%$ & $163.0 \%$ & $-42.7 \%$ & 0.62 & 0.09 & 0.82 \\
Mexico & $23.6 \%$ & $31.8 \%$ & $131.6 \%$ & $-61.1 \%$ & 0.88 & 0.47 & 0.93 \\
Peru & $52.2 \%$ & $60.7 \%$ & $223.5 \%$ & $-73.1 \%$ & 0.69 & 0.24 & 0.87 \\
U.S & $5.9 \%$ & $18.0 \%$ & $65.4 \%$ & $-49.3 \%$ & 1.00 & 1.00 & 1.00 \\
\hline \hline
\end{tabular}

Although a higher level of co-movements between the Latin American and the U.S. stock markets after the crisis is remarkable by itself, there is another phenomenon that might be more

\footnotetext{
${ }^{1}$ In this figure, S\&P return is measured on the right, and the vertical line marks January 2008, when the U.S. financial crisis started.
} 
Figure 1: The Latin American v.s The S\&P Returns

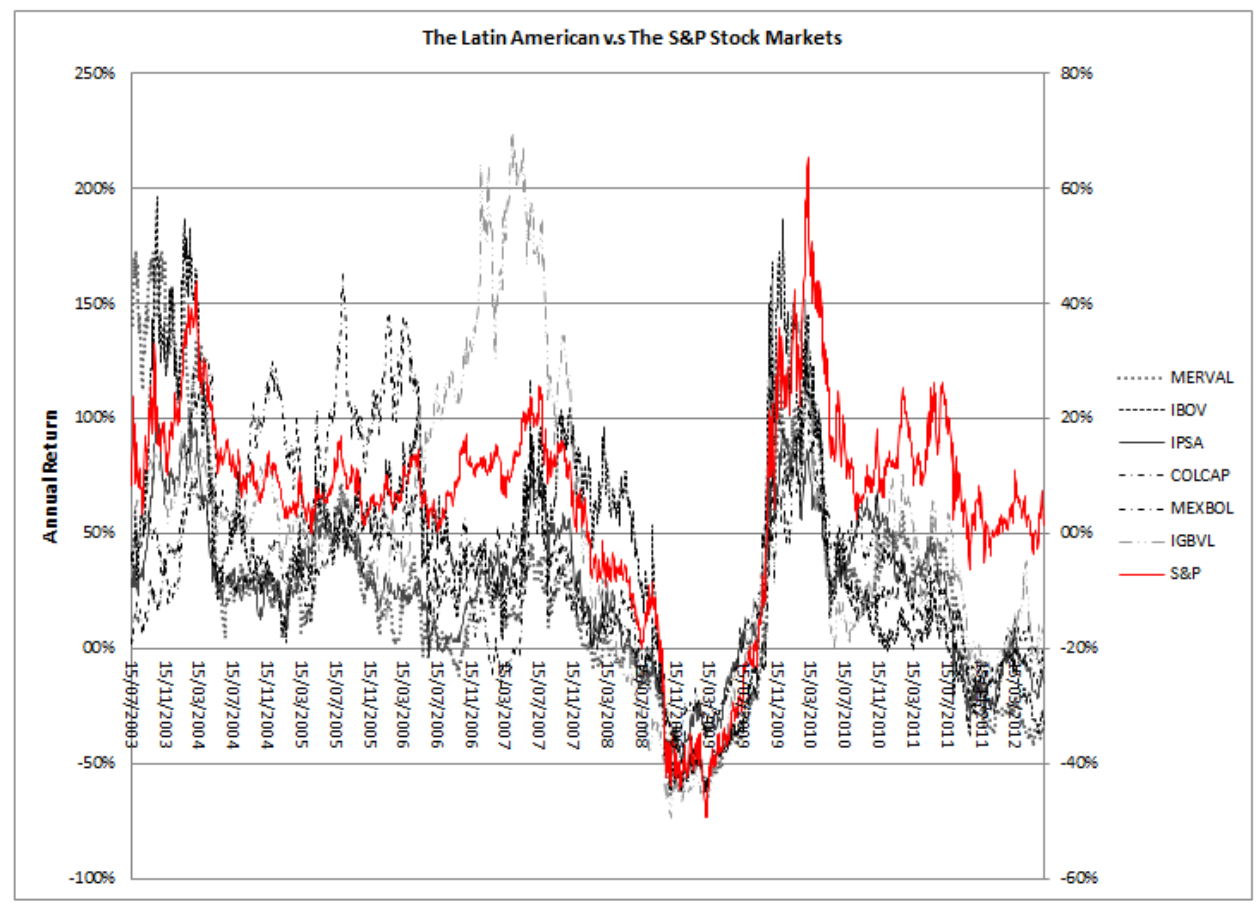

relevant for the Latin American region. After the crisis, there exists a higher level of correlation between the stock markets within the region. This fact can be observed in tables (2) and (3) where the matrix of correlation between the Latin American stock markets are shown for each period. We notice significant changes in the level of correlation. For example, consider the correlation coefficient between Colombia and Peru. From -0.64 in the period before the U.S. financial crises, the coefficient changes to 0.87 after the crisis. On explanation might be the creation of MILA. However, this stock market integration started at the end of 2010, and the correlation coefficient between these markets is 0.81 thereafter. Additionally, there is another remarkable change involving Peru, as the correlation coefficient between Argentina and Peru is -0.17 in the period 2003-2007, but after the crisis this coefficient goes up to 0.91. Finally, we have Argentina and Mexico, where the correlation is -0.11 in the former period, but 0.95 in the latter period.

After these preliminary descriptive exercises, we run some basic econometric models to get 
Table 2: Correlation Matrix: Annual rate of return Latin American Markets, 2003-2007.

\begin{tabular}{lcccccc}
\hline Country & Argentine & Brazil & Chile & Colombia & Mexico & Peru \\
\hline Argentine & 1.00 & 0.67 & 0.71 & 0.13 & -0.11 & -0.17 \\
Brazil & 0.67 & 1.00 & 0.75 & 0.17 & 0.23 & -0.13 \\
Chile & 0.71 & 0.75 & 1.00 & 0.12 & 0.26 & 0.08 \\
Colombia & 0.13 & 0.17 & 0.12 & 1.00 & 0.40 & -0.64 \\
Mexico & -0.11 & 0.23 & 0.26 & 0.40 & 1.00 & 0.29 \\
Peru & -0.17 & -0.13 & 0.08 & -0.64 & 0.29 & 1.00 \\
\hline \hline
\end{tabular}

Table 3: Correlation Matrix: Annual rate of return Latin American Markets, 2008-2012.

\begin{tabular}{lcccccc}
\hline Country & Argentine & Brazil & Chile & Colombia & Mexico & Peru \\
\hline Argentine & 1.00 & 0.82 & 0.93 & 0.93 & 0.95 & 0.91 \\
Brazil & 0.82 & 1.00 & 0.79 & 0.84 & 0.85 & 0.78 \\
Chile & 0.93 & 0.79 & 1.00 & 0.93 & 0.89 & 0.88 \\
Colombia & 0.93 & 0.84 & 0.93 & 1.00 & 0.93 & 0.87 \\
Mexico & 0.95 & 0.85 & 0.89 & 0.93 & 1.00 & 0.90 \\
Peru & 0.91 & 0.78 & 0.88 & 0.87 & 0.90 & 1.00 \\
\hline \hline
\end{tabular}

a better picture of the co-movements between the Latin American and S\&P stock indices. Specifically, we perform a regression analysis using the following specification:

$$
R_{L A, t}=\beta_{1}+\beta_{2} R_{U S, t}+v_{t}
$$

where $R_{L A, t}$ and $R_{U S, t}$ are the annual rate of return of the Latin American and U.S stock markets.

As we can see in Table (4), all parameters are statistically significant at $1 \%$. We observe that an increase of $1 \%$ in the $\mathrm{S} \& \mathrm{P}$ index implies a rise of $2.33 \%$ in the Peruvian index. On the other hand, Chile exhibits the lowest coefficient with 1.42. Additionally, the highest level of explanation of the U.S. stock market is associated with Mexico, whereas Colombia shows the lowest determination coefficient.

However, an estimation based on Ordinary Least Square gives a static picture of the comovements between these stock markets. In order to calculate the dynamic of the co-movements between the Latin American stock markets and the U.S. market, and analyse the effect of the 
Table 4: Parameter estimates of the model $R_{L A, t}=\beta_{1}+\beta_{2} R_{U S, t}+v_{t}$ : Annual rate of return, 2003-2012.

\begin{tabular}{lccc}
\hline Country & $\beta_{1}$ & $\beta_{2}$ & $R^{2}$ \\
\hline Argentine & $0.135^{*}$ & $2.087^{*}$ & 0.482 \\
& $(0.007)$ & $(0.040)$ & \\
Brazil & $0.277^{*}$ & $2.009^{*}$ & 0.489 \\
& $(0.008)$ & $(0.046)$ & \\
Chile & $0.181^{*}$ & $1.429^{*}$ & 0.654 \\
& $(0.004)$ & $(0.023)$ & \\
Colombia & $0.333^{*}$ & $1.560^{*}$ & 0.378 \\
& $(0.008)$ & $(0.045)$ & \\
Mexico & $0.145^{*}$ & $1.545^{*}$ & 0.766 \\
& $(0.003)$ & $(0.019)$ & \\
Peru & $0.384^{*}$ & $2.335^{*}$ & 0.482 \\
& $(0.010)$ & $(0.055)$ & \\
\hline * Statistically significant at 1\%. \\
Standard deviation in parenthesis. \\
\hline \hline
\end{tabular}

financial crisis on these co-movements, we estimate the following model:

$$
R_{L A, t}=\beta_{1, t}+\beta_{2, t} R_{U S, t}+v_{t} .
$$

We set as prior distribution for the state vector a Gaussian distribution, that is $\left[\begin{array}{c}\beta_{1,0} \\ \beta_{2,0}\end{array}\right] \sim$ $\mathcal{N}\left(\left[\begin{array}{l}0 \\ 0\end{array}\right],\left[\begin{array}{cc}1 e+07 & 0 \\ 0 & 1 e+07\end{array}\right]\right)$

We estimate $\sigma_{v}^{2}, \sigma_{w_{1}}^{2}$ and $\sigma_{w_{2}}^{2}$ using Maximum Likelihood as proposed in Section (2). ${ }^{2}$

Figure (2) shows that there is a high degree of variability between the co-movements in the Latin American stock market and the S\&P index in the period 2004-2012. ${ }^{3}$ This variability is evident between countries and also within each country. We can observe that the Brazilian market has the highest sensitivity to the U.S. market with a mean of 1.91, but also the highest

\footnotetext{
${ }^{2}$ All calculations are done using R package (R Development Core Team, 2011), and specifically, the dlm library (Petriss, 2010).

${ }^{3}$ We omit the first 100 estimations because of dependence of the prior distribution.
} 
volatility with a standard deviation of 0.69 . On the other hand, Chile has the smallest volatility with a standard deviation of 0.22. Additionally, Colombia, Peru and Chile show the smallest sensitivity to the S\&P index (see Table 5). The case of Peru is remarkable because when using OLS this country has the highest coefficient (2.33).

Figure 2: Dynamic Beta: The Latin American Stock Markets

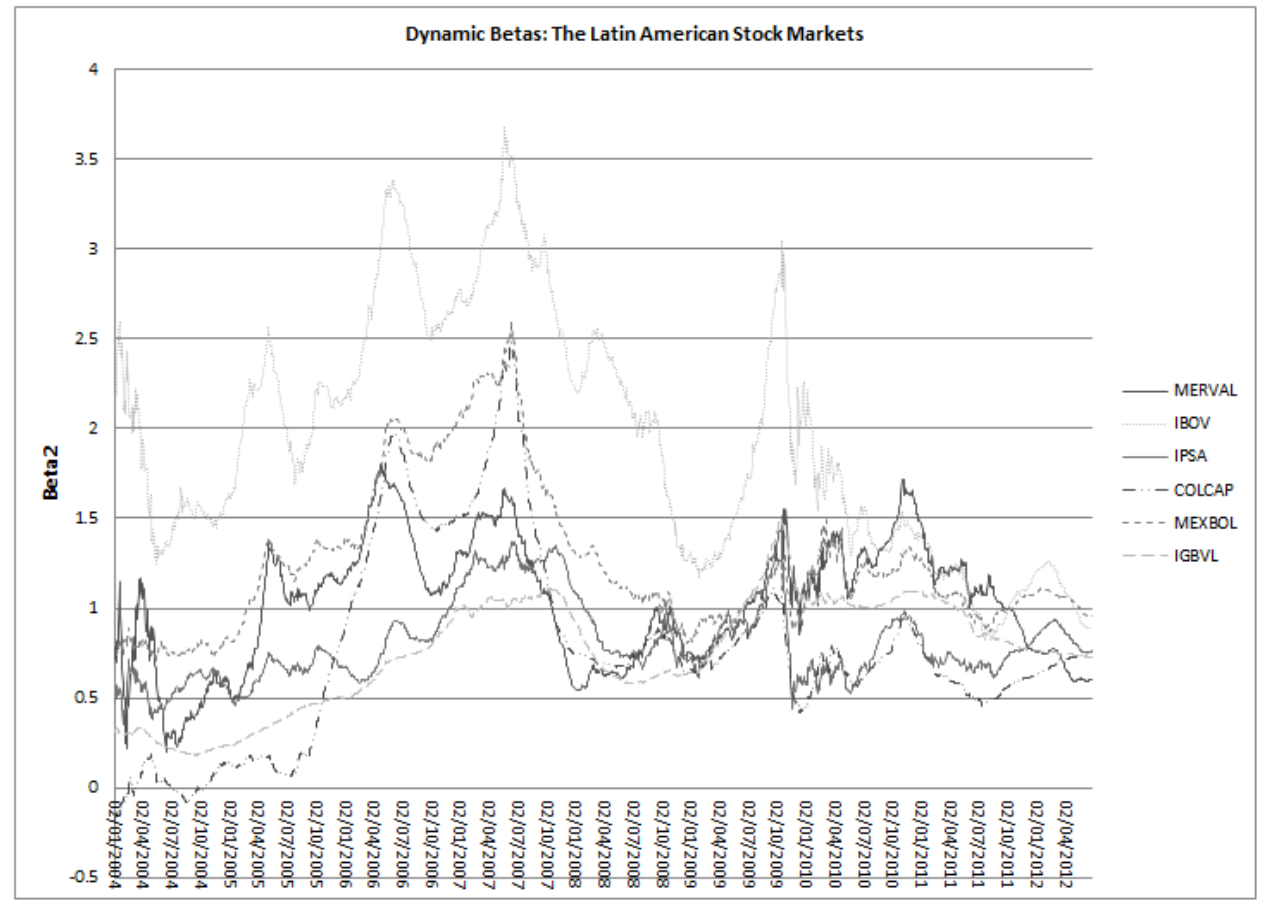

Regarding the pattern of the co-movements between the Latin American and U.S. stock markets before the financial crisis in 2008, we find an upward trend of the sensitivity of these markets between January 2004 and June 2007, as displayed in Figure (2). It must be taken into consideration that in this month, there were a lot of news related to vulnerability of financial markets. After this period, there is a downward trend until January 2008, when the financial crisis in United States erupted. After this event, we notice oscillations of the co-movements between the Latin American stock markets and the S\&P index.

However, we observe an undeniable empirical fact, less volatility in the level of sensitivity 
Table 5: Descriptive statistics: Dynamic co-movements between Latin American and USA stock markets, 2003-2012.

\begin{tabular}{lcccc}
\hline Country & Mean & Standard Deviation & Maximum & Minimum \\
\hline Argentine & 1.01 & 0.34 & 1.80 & 0.20 \\
Brazil & 1.91 & 0.69 & 3.68 & -0.01 \\
Chile & 0.79 & 0.22 & 1.51 & 0.35 \\
Colombia & 0.72 & 0.56 & 2.58 & -0.18 \\
Mexico & 1.22 & 0.42 & 2.59 & 0.42 \\
Peru & 0.72 & 0.31 & 1.50 & 0.16 \\
\hline \hline
\end{tabular}

of the different Latin American stock markets and the U.S. market. As can be seen in Table (6), there exists a decrease in the standard deviation of $\beta_{2, t}$. For instance, we observe Peru with a standard deviation of 0.32 in the first period, and this volatility drops to 0.20 in the second period. Colombia had the most significant decrease in the level of volatility. The country had a standard deviation of 0.78 in the period 2004-2007 in its sensitivity with respect to U.S. stock market, but the volatility reduces to 0.13 in 2008-2012, which is one of the lowest.

Table 6: Volatility of sensitivity $\left(\beta_{2, t}\right)$ : Latin American Markets, 2004-2012.

\begin{tabular}{lcccccc}
\hline Country & Argentine & Brazil & Chile & Colombia & Mexico & Peru \\
\hline $2004-2007$ & 0.39 & 0.70 & 0.28 & 0.78 & 0.57 & 0.32 \\
$2008-2012$ & 0.28 & 0.50 & 0.15 & 0.13 & 0.13 & 0.20 \\
\hline \hline
\end{tabular}

The most important aspect of these patterns consists in a convergence of the level of sensitivity of Latin American stock markets with the U.S. stock market after the financial crisis. This is not just a phenomenon that is present in each country through time, but rather a phenomenon that affects each country in a consistent way. For example, the country with the highest average $\beta_{2, t}$ in the period 2004-2007 is Brazil with 2.25, while Peru had the lowest average $\beta_{2, t}$ with 0.56. In the second period, 2008-2012, Brazil's $\beta_{2, t}$ decreases to 0.64, whereas Peru had an increase of 0.33. These changes imply that the average $\beta_{2, t}$ of Peru and Brazil in the second period are 0.89 and 1.57 , respectively. We find a reduction in the highest gap of these parameters of almost one. 
This convergence can also be observed in Figure (3), plotting the standard deviation between the sensitivity of each Latin American stock market. We note a significant reduction in this standard deviation. In the first period, this measure has a mean of 0.67 , whereas in the period 2008-2012 this mean decreases to 0.35. A possible explanation is that in recent years there have been increased interactions between international financial markets due to a higher level of economic integration, investment, capital mobility, and a greater flow of information. This translates into the impact that events occurring in some markets are transmitted to others between different regions in the world. Studies have showed evidence about co-movements and volatility transmission among financial markets in Latin America and U.S. financial market (Sakthivel et al., 2012; Weber, 2013). Presumably, this phenomenon was accelerated by the financial crisis in the U.S. market.

Figure 3: Standard Deviation: The Latin American sensitivity to the S\&P index

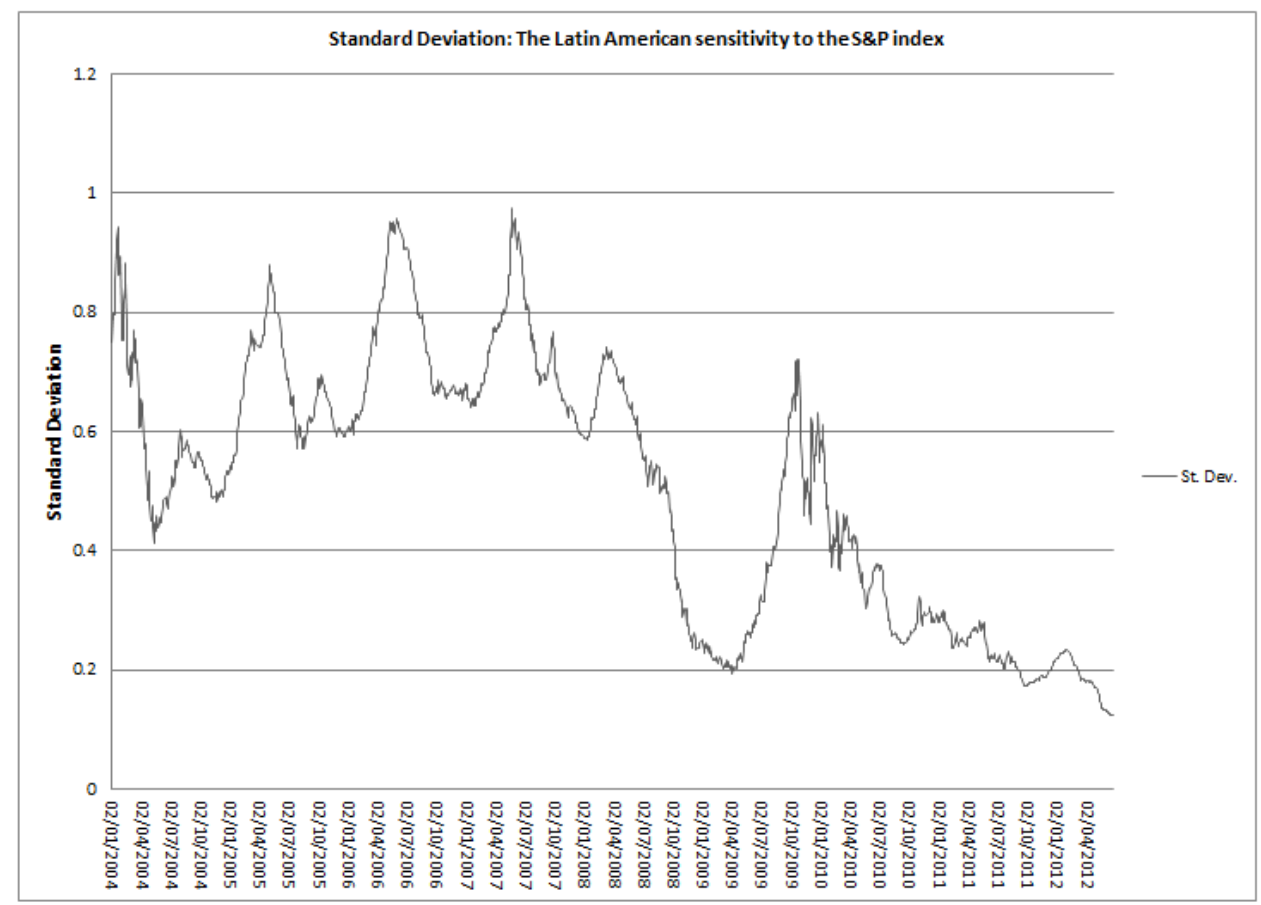




\section{Conclusions}

Convergence in stock markets is a gradual phenomenon in a world that is characterised by a stronger financial integration, and it seems that the Latin American stock markets are not an exception to this pattern. However, there are some specific events that might accelerate this phenomenon. Specifically, it appears as if the financial crisis in U.S. caused a synchronization between the Latin American stock markets. We show that there exists a higher level of correlation between the Latin American stock markets after the U.S. financial crisis in 2008. Additionally, we find that the sensitivity of the Latin American stock markets to the U.S. market moves in a consistent way that implies convergence of this region with regard to the S\&P index. This kind of homogeneous reaction might help to accelerate the process of financial integration that started in the region since 2010. 


\section{References}

Allen, F., Gu, X., and Kowaslewski, O. (2012). Financial crisis, structure and reform. Journal of Banking and Finance, 36:2960-2973.

Bracker, K. and Koch, P. (1999). Economic determinants of the correlation structure across international equity markets. Journal of Economics and Business, 51:443-471.

Chen, G., Firth, M., and O.Rui (2002). Stock market linkages: Evidence from Latin America. Journal of Banking \& Finance, 26:1113-1141.

Fayyad, A. and Daly, K. (2011). International transmission of stock returns: Mean and volatility spillover effects in the emerging markets. International Research Journal of Finance and Economics, 67:1-67.

Lee, B. and Rui, O. (2004). Information transmission between the NASDAG and Asian Second Board Markets. Journal of Banking and Finance, 28:1637-1670.

Liow, K. (2009). Co-movements and correlations across Asian securitized real state and stock markets. Working paper, pages 1-53.

Petris, G., Petrone, S., and Campagnoli, P. (2007). Dynamic Linear Models with R. Springer.

Petriss, G. (2010). An R package for dynamic linear models. Journal of Statistical Software, $36(12): 1-16$.

R Development Core Team (2011). R: A Language and Environment for Statistical Computing. R. Version 2.13.0. R Foundation for Statistical Computing, Vienna, Austria. ISBN 3-900051$07-0$.

Sakthivel, P., Bodkhe, N., and Kamaiah, B. (2012). Correlation and volatility transmission across international stock markets: A bivariate garch analysis. International Journal of Economics and Finance, 4(3):253-264.

Weber, E. (2013). Simultaneous stochastic volatility transmission across American equity markets. The Quarterly Review of Economics and Finance, 53(1):53-60. 